\title{
ODOUR REDUCTION STRATEGIES FOR BIOSOLIDS PRODUCED FROM A WESTERN AUSTRALIAN WASTEWATER TREATMENT PLANT: RESULTS FROM PHASE I LABORATORY TRIALS
}

\author{
YOLANTA GRUCHLIK ${ }^{\#, 1}$, ANNA HEITZ ${ }^{2}$, CYNTHIA JOLL ${ }^{1}$, HANNA DRIESSEN ${ }^{1}$, LISE \\ FOUCHE $^{1}$, NANCY PENNEY ${ }^{3}$ AND JEFFREY W.A. CHARROIS ${ }^{1}$
}

\author{
${ }^{1}$ Curtin Water Quality Research Centre (CWQRC), Department of Chemistry, Curtin University, \\ Resources and Chemistry Precinct, GPO Box U1987, Perth, Western Australia, 6845 \\ ${ }^{2}$ Department of Civil Engineering, Curtin University, GPO Box U1987, Perth, Western Australia, 6845 \\ ${ }^{3}$ Water Corporation of Western Australia, 629 Newcastle Street, Leederville, WA 6007 \\ ${ }^{\#}$ Corresponding author email: Y.Gruchlik@ curtin.edu.au
}

\begin{abstract}
This study investigated sources of odours from biosolids produced from a Western Australian Wastewater Treatment Plant (WWTP) and examined possible strategies for odour reduction, specifically chemical additions and reduction of centrifuge speed on a laboratory scale. To identify the odorous compounds and assess the effectiveness of the odour reduction measures trialled in this study, headspace solid-phase microextraction gas chromatography-mass spectrometry (HS SPME-GC-MS) methods were developed. The target odour compounds included volatile sulphur compounds (e.g. dimethyl sulphide, dimethyl disulphide and dimethyl trisulphide) and other volatile organic compounds (e.g. toluene, ethylbenzene, styrene, $p$-cresol, indole and skatole). In our laboratory trials, aluminium sulphate added to anaerobically digested sludge prior to dewatering offered the best odour reduction strategy amongst the options that were investigated, resulting in approximately $40 \%$ reduction in the maximum concentration of the total volatile organic sulphur compounds (TVOSC), relative to control.
\end{abstract}

Keywords: biosolids, HS SPME-GC-MS, odour, odour reduction, volatile sulphur compounds,

\section{INTRODUCTION}

The production of odours during biosolids storage and land application is a significant problem for wastewater utilities and can have a negative impact on beneficial re-use programs. As such, considerable research has been carried out to understand the causes of odour formation in biosolids and to develop management strategies to minimise these odours (Adams et al., 2008). Odour production in biosolids is a complex process influenced by several factors including, but not limited to, process variables within anaerobic digestion and dewatering processes, as well as the relationships between odours and the concentrations of odorants (Adams et al., 2008). Due to differences in biosolids characteristics, treatment processes and operating conditions associated with different wastewater treatment plants, the chosen odour reduction strategies need to be based on the site-specific conditions at each wastewater treatment plant (WWTP). In most cases, laboratory and/or pilot-scale trials would be required to find the most suitable odour reduction strategy (Adams et al., 2008).

Compounds associated with odours from biosolids facilities include: volatile organic sulphur compounds (VOSCs) such as: methanethiol (MT), dimethyl sulphide (DMS), dimethyl disulphide 
(DMDS), dimethyl trisulphide (DMTS), as well as inorganic sulphur compounds such as hydrogen sulphide $\left(\mathrm{H}_{2} \mathrm{~S}\right)$ (Adams et al., 2008; Higgins et al., 2008). Nitrogenous compounds such as trimethylamine (TMA), ammonia, and volatile fatty acids (VFA) can also be potential sources of odour (Higgins et al., 2008). Odorous volatile aromatic compounds (OVACs) such as toluene, ethylbenzene, styrene, $p$-cresol, indole and skatole have been identified in headspace samples from stored biosolids (Chen et al., 2006).

To assess the effectiveness of biosolids odour reduction strategies, appropriate sampling and analytical techniques are required to measure accurately the odorous compounds present in the biosolids cake. Glindemann et al. (2006) used a static headspace method for the analysis of odorous gases from dewatered sludge cakes in the laboratory. This method utilised gas-tight bottles for incubating biosolids and involved manual sampling and injection of the headspace gases into the GC inlet using a gas-tight syringe (Glindemann et al., 2006). Although this method has been reported to be representative of the biosolids storage pile interior, easy to use and highly reproducible (Glindemann $e t$ al., 2006), manual injections of the headspace gases into the GC inlet are time consuming and laborious, and limited to analysis of only a few samples (Gruchlik et al., 2012). Solid-phase microextraction (SPME) coupled with GC-MS has also been used for the analysis of odorous compounds in several biosolids projects (e.g. Turkmen, et al., 2004; Kim et al., 2005). However, many of these methods used complicated apparatus for the calibration and sampling of the gaseous odorants and involved manual injections of the SPME fibre into the GC injector.

In this study we used SPME-GC-MS for the analysis of odorous compounds in the headspace of wet biosolids. In this method biosolids samples were analysed as "aqueous" samples, which does not require any complex sampling equipment, is reproducible, and the analysis is fully automated, allowing for a higher throughput of samples (Gruchlik et al., 2012).

The main objectives of this study were to: (a) develop analytical methods to identify the chemical compounds responsible for the odour in biosolids from our test site, (b) assess the effectiveness of trialled odour reduction measures and (c) determine the most suitable odour reduction strategy for biosolids produced at our test site.

\section{METHODOLGY}

Anaerobically digested sludge (10 L, dry solids (DS) 3.7\%, sludge retention time (SRT) 19 days) and plant dewatered biosolids cake (1 kg, DS 16.9\%) were obtained from a Perth metropolitan WWTP, which uses activated sludge and sequencing batch reactors (SBR), egg-shaped mesophilic anaerobic digestion and centrifuge dewatering. The samples were packed in coolers and transported to the laboratory for use in bench-scale trials (chemical additions and reduction of centrifuge speed). The same batch of anaerobically digested sludge and plant dewatered biosolids cake was used for all chemical addition and centrifuge-speed trials described in this study. The sludge and biosolids samples were stored in a cool room at $4{ }^{\circ} \mathrm{C}$. Polymer used for dewatering was a powder polymer FO4800SSH from SNF Pty Ltd (supplied by Water Corporation) with a molecular weight of approximately 8 million and a charge density of $80 \%$. The polymer was dosed as a $0.3 \%(\mathrm{w} / \mathrm{v})$ aqueous solution and the polymer dose was based on the average amount used at our test site and adjusted accordingly to the lab-scale experiments. Aluminium sulphate (alum; $\mathrm{Al}_{2}\left(\mathrm{SO}_{4}\right)_{3} \cdot 14 \mathrm{H}_{2} \mathrm{O}$ ) was used as a $56 \%$ w/v aqueous solution. Polyaluminium chloride (PAC23 from Orica) was used as a $23 \% \mathrm{w} / \mathrm{v}$ solution as aluminium oxide. Ferric chloride was used as a $36 \%$ w/v aqueous solution, prepared in-house from analytical grade ferric chloride (Sigma-Aldrich).

\section{Laboratory Scale Dewatering}

We used a laboratory scale dewatering procedure, previously developed, using a laboratory centrifuge (Heraeus Multifuge 3S with a maximum rotational radius of $18.2 \mathrm{~cm}$ ) (Gruchlik et al., 2012). In order 
to simulate the dewatering conditions used at our test site the centrifuge was operated at $3850 \mathrm{rpm}$ for 20 minutes. The resulting cake was then processed through a manual food mincer (Avanti food mincer \#8) which pushed the cake through a "scroll-conveyor", followed by extrusion through several openings. The lab dewatered biosolids cake had a similar texture and odour to the plant dewatered sample. The solids content of the lab dewatered cake was comparable to that of the plant dewatered cake.

\section{Chemical addition to digested sludge prior to dewatering}

Individual samples of anaerobically digested sludge (approximately $800 \mathrm{~g}$ each) in $1 \mathrm{~L}$ glass beakers were treated with alum, polyaluminium chloride and ferric chloride at doses of $2 \%$ and $4 \%$ of metal on a dry weight basis, followed by addition of polymer. The samples were mixed using a jar tester. The mixing regime used was based on that reported by Higgins (2010) and is shown in Figure 1. A control sample, with no chemical addition was also prepared. The samples were dewatered using the dewatering procedure developed in-house. The resulting biosolids cake samples (approximately $200 \mathrm{~g}$ ) were incubated at room temperature in $1 \mathrm{~L}$ Schott bottles. The samples were wrapped in aluminium foil to protect them from light and were monitored for evolution of volatile sulphur compounds (DMS, EMS (ethyl methyl sulphide), DMDS, DEDS (diethyl disulphide) and DMTS) by HS SPME-GC-MS every few days over a period of 20 days. Samples were also analysed for the production of OVACs (toluene, ethylbenzene, styrene, $p$-cresol, indole, and skatole) by HS SPME-GC-MS weekly over a period of 37 days.

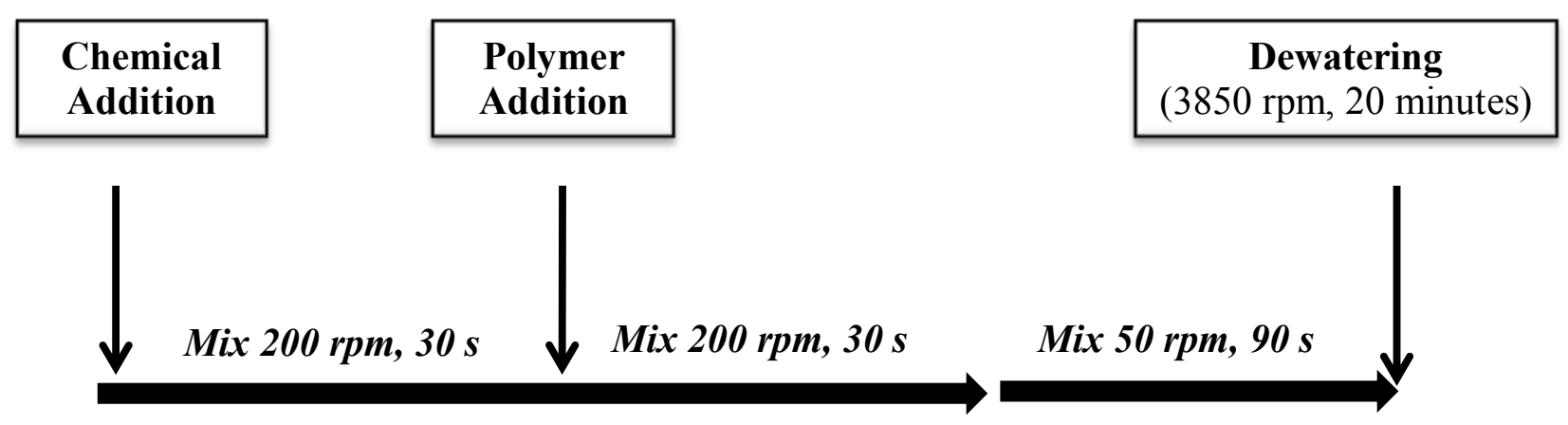

Figure 1. The mixing regime used in lab scale trials of chemical addition to digested sludge (adapted from Higgins, 2010; Gruchlik et al., 2012).

\section{Chemical addition to plant dewatered cake}

Samples of the plant dewatered biosolids (approximately $85 \mathrm{~g}$ ) in $400 \mathrm{~mL}$ glass beakers were treated with aluminium sulphate hydrate at doses of $2 \%$ and $4 \%$ of metal on dry weight basis and mixed manually with a stainless steel spatula for approximately 2 minutes. A control sample (no chemical addition) was prepared in the same way. Cake samples were incubated at room temperature in $250 \mathrm{~mL}$ Schott bottles. Samples were wrapped in aluminium foil to protect them from light and monitored for evolution of volatile sulphur compounds by HS SPME-GC-MS every few days over a period of 14 days. Samples were also analysed for the production of OVACs by HS SPME-GC-MS weekly over a period of 16 days.

\section{Centrifuge-speed trials}

Samples of untreated (i.e. no chemical addition except for polymer) anaerobically digested sludge (approximately $600 \mathrm{~g}$ each) were dewatered at $3850 \mathrm{rpm}$ (control speed), $3460 \mathrm{rpm}$ (10\% reduction in speed, relative to control) and $3080 \mathrm{rpm}$ (20\% reduction in speed, relative to control) using the dewatering procedure developed in-house. The resulting biosolids cake samples (approximately $190 \mathrm{~g}$ ) were incubated at room temperature in $500 \mathrm{~mL}$ Schott bottles. Samples were wrapped in aluminium foil and were monitored for evolution of volatile sulphur compounds by HS SPME-GC-MS every few 
days for a period of 20 days. Samples were also analysed for the production of OVACs by HS SPMEGC-MS weekly for a period of 23 days.

\section{HS SPME-GC-MS procedure for the analysis of sulphur compounds}

Sulphur compounds (DMS, EMS, DMDS, DEDS and DMTS) were analysed by headspace SPME using a 50/30 $\mu \mathrm{m}$ DVB-CAR-PDMS (divinylbenzene-carboxen-polydimethylsiloxane) fibre, followed by GC-MS analysis. SPME was performed using a Gerstel MPS2 Autosampler interfaced with a Hewlett Packard 6890N GC and a Hewlett Packard 5973 Network Mass Selective Detector. A sample of biosolids cake $(50-80 \mathrm{mg})$ was placed into a Teflon-lined screw cap vial $(20 \mathrm{~mL})$ and $10 \mathrm{~mL}$ of a $500 \mathrm{ng} / \mathrm{L}$ DMDS- $d_{6}$ internal standard solution in MilliQ water was added, followed by anhydrous sodium sulphate $(3 \mathrm{~g})$. The SPME fibre was introduced into the headspace of the vials and extraction was carried out for 10 minutes at $40{ }^{\circ} \mathrm{C}$. The fibre was then desorbed at $230{ }^{\circ} \mathrm{C}$ for 4 minutes in the injector port of the $\mathrm{GC}$, while analytes were simultaneously cryofocused on the $\mathrm{GC}$ column at $0{ }^{\circ} \mathrm{C}$. The oven temperature program was set up as follows: $0{ }^{\circ} \mathrm{C}$ for $2.00 \mathrm{~min}$, increased at $5{ }^{\circ} \mathrm{C} / \mathrm{min}$ to 35 ${ }^{\circ} \mathrm{C}$ then increased at $15^{\circ} \mathrm{C} / \mathrm{min}$ to $315{ }^{\circ} \mathrm{C}$ and held at $315{ }^{\circ} \mathrm{C}$ for $10 \mathrm{~min}$ (run time $=37.67 \mathrm{~min}$ ). GC separation of sulphur compounds was carried out using helium as the carrier gas at $1.0 \mathrm{~mL} / \mathrm{min}$, and a $30 \mathrm{~m}$ x $0.25 \mathrm{~mm}$ x $1 \mu \mathrm{m}$ ZB-5MS (Phenomenex®) capillary column. The mass spectrometer (MS) was operated in selected ion monitoring (SIM) mode and for each sulphur compound, the most abundant ion was used for quantitation and $1-2$ characteristic $m / z$ ions were selected for MS confirmation. Samples were analysed against standards of the pure compounds with deuterated DMDS (DMDS- $d_{6}$ ) as an internal standard.

\section{HS SPME-GC-MS procedure for the analysis of OVACs}

The OVACs (toluene, ethylbenzene, styrene, $p$-cresol, indole and skatole) were analysed using a similar procedure to that described above for the sulphur compounds except that a $65 \mu \mathrm{m}$ PDMS-DVB (polydimethylsiloxane-divinylbenzene) fibre was used, and extraction was carried out for 30 minutes at $60{ }^{\circ} \mathrm{C}$. The fibre was desorbed at $250{ }^{\circ} \mathrm{C}$ for 5 minutes in the injector port of the GC and the analytes were not cryofocused. The oven temperature program was set up as follows: $40{ }^{\circ} \mathrm{C}$ for $5.00 \mathrm{~min}$, increased at $20{ }^{\circ} \mathrm{C} / \mathrm{min}$ to $200{ }^{\circ} \mathrm{C}$, then increased at $10{ }^{\circ} \mathrm{C} / \mathrm{min}$ to $280{ }^{\circ} \mathrm{C}$ and then increased at 20 ${ }^{\circ} \mathrm{C} / \mathrm{min}$ to $300{ }^{\circ} \mathrm{C}$ and held at $300{ }^{\circ} \mathrm{C}$ for $10 \mathrm{~min}$ (run time $=32.00 \mathrm{~min}$ ). Samples were analysed against standards of pure compounds using deuterated ethylbenzene (ethylbenzene- $d_{10}$ ) as an internal standard.

\section{Analysis of biosolids samples from the odour reduction trials}

Odours from the biosolids samples obtained during odour reduction trials were assessed in terms of the concentration of the total volatile organic sulphur compounds (TVOSC), measured as the sum of the DMS, DMDS and DMTS concentrations present in the biosolids samples and compared with a control sample. Odour reduction (or increase) was considered to be the reduction (or increase) in the maximum TVOSC concentration relative to the control sample.

\section{RESULTS AND DISCUSSION}

\section{Validation and optimisation of the HS SPME/GC-MS methods for the analysis of sulphur compounds and OVACs}

GC-MS conditions (oven temperature, injector temperature, flow rate and MS parameters) and HS SPME parameters (fibre type, extraction temperature and time, and desorption conditions) for the analysis of sulphur compounds and OVACs were optimised, in order to achieve good baseline separation of analytes and maximise sensitivity and response, while minimising analyte degradation and carry-over (Gruchlik et al., 2012). 


\section{Degradation of analytes}

Certain sulphur compounds such as mercaptans and dimethylpolysulphides (e.g. DMDS) can undergo degradation under certain conditions (Haberhauer-Troyer et al., 1999; Kristiana et al., 2010). Under our method conditions, methanethiol was oxidised to DMDS (major peak) and DMTS (minor peak); and ethanethiol (ET) was oxidised to DEDS (major) with only a very minor peak visible for ET. Analysis of a solution containing a mixture of MT and ET showed major peaks for DEDS and the "scrambled" compound methyl ethyl disulphide (MEDS) formed as a result of a reaction between MT and ET, as well as smaller peaks for DMDS and DMTS. No degradation products were observed for any of the other analytes (Gruchlik et al., 2012). Since MT and ET were too reactive under our method conditions, they were excluded from the mixed standard solution. It was assumed that any MT present in the biosolids would be transformed to DMDS and DMTS. Similarly, any ET present in the biosolids would be converted to DEDS.

\section{Odorous compounds identified in biosolids samples from our test site}

The main odour compounds identified in fresh biosolids samples from our test site included: DMS, DMDS and DMTS. Indole and skatole were identified in older biosolids samples that were stored at room temperature for a few months and exhibited a strong faecal/nauseating odour. These compounds were not detected in the fresh biosolids samples. This finding is consistent with previous reports that one of the major sources of odours during the first 1-2 weeks of biosolids storage is due to the production of VOSCs by microbial degradation of sulphur-containing amino acids, while the OVACs start to accumulate only after VOSCs have been depleted (Chen, et al., 2006). Other types of compounds which were tentatively identified based on their mass spectra and/or library matches, but not confirmed with authentic analytical standards, included: various long chain aliphatic hydrocarbons, terpenes, alkyl benzenes and other aromatic compounds.

\section{Chemical addition as an odour reduction strategy}

Previous research has shown that many of the odour compounds associated with biosolids (e.g. MT, DMS, DMDS) can be formed during microbial degradation of bioavailable protein (Higgins et al., 2006). Thus, reducing the amount of bioavailable protein in dewatered biosolids may reduce the formation of odour causing compounds (Higgins, 2010). Trivalent metals such as, aluminium (Al) and iron $(\mathrm{Fe})$ can bind labile proteins within the floc structure of sludge/biosolids (Subramanian et al., 2005). Therefore, addition of aluminium or iron(III) salts to biosolids could reduce the amount of bioavailable protein thereby reducing the production of odorous compounds (Higgins, 2010). In a series of laboratory scale experiments, researchers found that addition of alum to digested sludge prior to dewatering significantly reduced the production of odour compounds (Adams et al., 2008; Higgins, 2010). In some cases up to $80 \%$ reductions of the peak TVOSC concentrations were observed for aluminium doses as low as $0.5 \%$ (dry mass - mass basis) (Adams et al., 2008).

The chemicals could be added during conditioning and dewatering or directly to the dewatered cake: it was found that adding metal salts directly to the cake gave better TVOSC reductions compared to adding the salts during the conditioning and dewatering step. However, chemical addition to the cake also resulted in a greater reduction in the $\mathrm{pH}$ of the cake to levels below those desirable for land application (Higgins, 2010).

In this study, laboratory scale experiments of addition of aluminium sulphate, polyaluminium chloride and ferric chloride to digested sludge prior to dewatering at doses of $0 \%$ (control), $2 \%$ and $4 \%$ of metal on dry weight basis were conducted. In all of our experiments the primary coagulants were added prior to adding the polymer (Figure 1). The addition of aluminium sulphate to plant dewatered biosolids cakes was also investigated. 


\section{Chemical addition to digested sludge prior to dewatering}

A $37 \%$ reduction of the maximum TVOSC concentration was observed for an aluminium dose of $2 \%$, while a $4 \% \mathrm{Al}$ dose resulted in a $40 \%$ reduction of the maximum TVOSC concentration, relative to the control sample (Figure 2a).
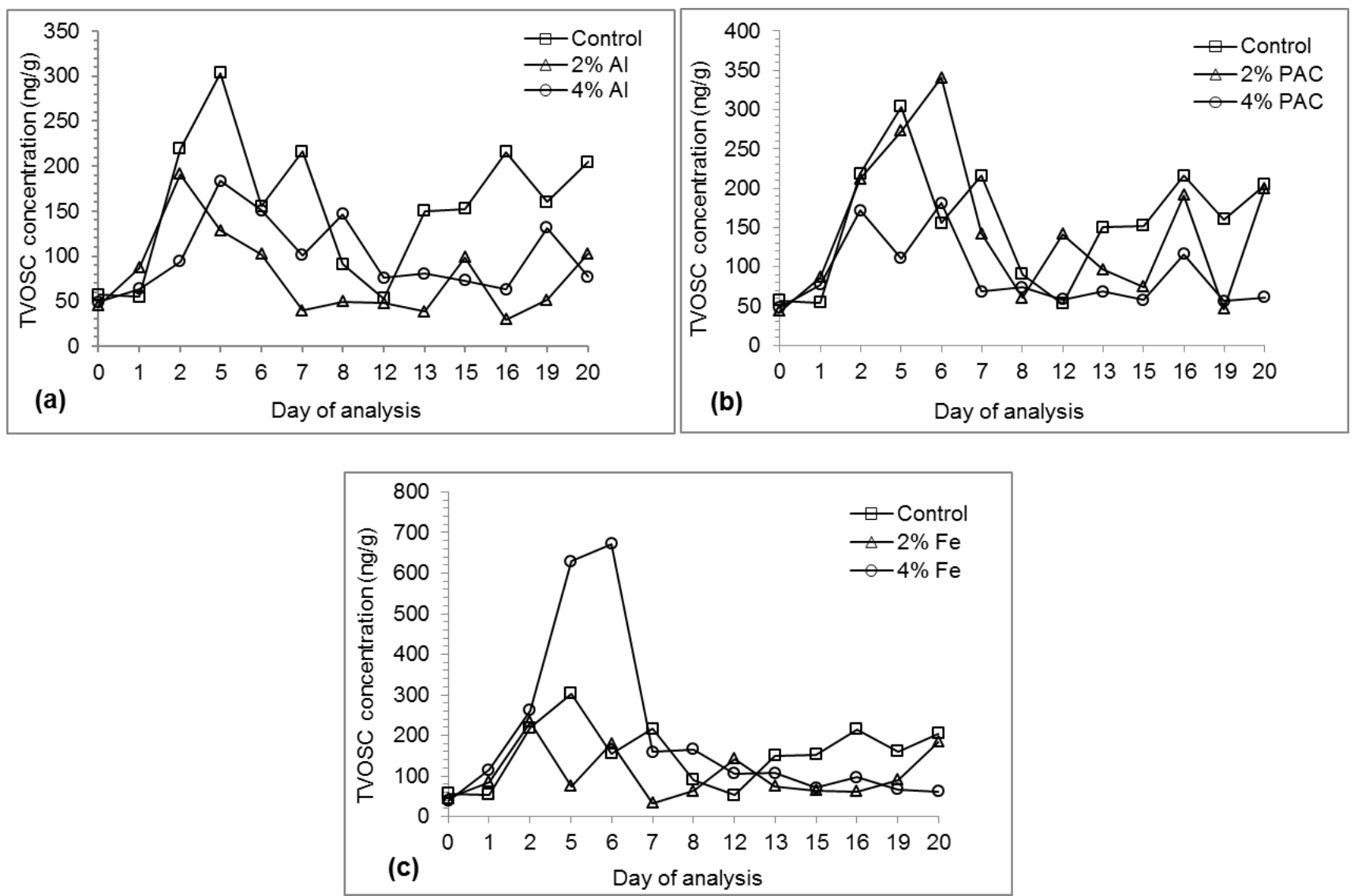

Figure 2. TVOSC (total volatile organic sulphur compounds) production in biosolids cakes obtained from laboratory scale trials of: (a) aluminium sulphate (Al) addition; (b) polyaluminium chloride (PAC) addition and (c) ferric chloride $(\mathrm{Fe})$ addition to digested sludge prior to dewatering. (Note:

TVOSC $=$ the sum of DMS, DMDS and DMTS concentrations.)

The odour reductions observed in our laboratory trials were lower than those reported by the Water Environment Research Foundation (WERF) research team (Adams et al., 2008). In one of their laboratory trials, using anaerobically digested sludge from a WWTP located in the east coast of the USA, the WERF researchers observed that the lowest alum dosage examined ( $0.5 \%$ as aluminium) reduced the maximum TVOSC concentration by approximately $83 \%$, while a $2 \% \mathrm{Al}$ dose resulted in approximately $90 \%$ reduction in the maximum TVOSC concentration (Adams et al., 2008). The observed differences in the odour reductions obtained in our laboratory trials and those reported by the WERF research team may be due to a number of factors such as, differences in the sludge and biosolids characteristics, due to treatment processes and operating conditions associated with different WWTPs (e.g. sludge treatment upstream of anaerobic digestion, digestion conditions and SRT), type of polymer used, chemical contact time, mixing, shear and interactions between the metal and polymer (Adams et al., 2008; Gruchlik et al., 2012).

Addition of iron at the $2 \%$ dose resulted in only a slight decrease $(23 \%)$ in the maximum TVOSC concentration, while addition of iron at the $4 \%$ dose resulted in a 50\% increase in the maximum TVOSC concentration, relative to control (Figure 2c). It is unclear at this stage why the $4 \%$ iron dose resulted in such a large increase in the TVOSC concentration in our laboratory trials. Previously reported results with respect to iron addition as means of odour reduction have been somewhat 
inconsistent and contradictory, and dependent on the sludge properties and location of iron addition (Adams et al., 2008; Higgins, 2010; Novak and Park, 2010).

Comparison of TVOSC profiles in Figure 2 (a-c) showed that in all three cases the TVOSC concentrations reached a maximum within the first week of incubation and then decreased, which was consistent with previously reported research (Adams et al., 2008).

\section{Chemical addition to plant dewatered cake}

In our laboratory trials, a $2 \%$ dose of aluminium sulphate (based on aluminium) resulted in a $24 \%$ increase in peak TVOSC concentration, while addition of $4 \%$ aluminium sulphate resulted in approximately $70 \%$ decrease in peak TVOSC concentration, relative to the control sample. However, the $\mathrm{pH}$ of the cake treated with $4 \%$ aluminium sulphate was also significantly reduced $(\mathrm{pH} 4.2)$ to levels that may not be suitable for land application. Therefore to overcome the problem of low $\mathrm{pH}$, lime would need to be incorporated into the biosolids prior to land application to raise the $\mathrm{pH}(6-8)$ to a suitable level for land application. These results are consistent with previously reported data (Higgins 2010).

\section{Centrifuge-speed trials}

It has been reported that odour emissions from cakes dewatered by high-solids centrifuges were higher than cakes dewatered by other means (Adams et al., 2008). This was attributed to the high shear imparted on biosolids during centrifuge dewatering, as a result of higher centrifuge speeds and/or torque conditions. The shear created by centrifugation may increase the amount of bioavailable protein for odour production. Therefore reducing the centrifuge speed and/or torque can reduce the amount of shear imparted on biosolids, thereby reducing the odour of the dewatered cake (Adams et al., 2008). In a full-scale test, a $10 \%$ reduction in centrifuge bowl speed on one high-solids centrifuge resulted in $20 \%$ reduction of TVOSC emissions from dewatered cake with no observed reduction in cake solids concentration (Adams et al., 2008).

In this study we investigated reduction of centrifuge speed as means of odour reduction on a laboratory scale. Three centrifuge speeds were investigated: $3850 \mathrm{rpm}$ (control speed), $3460 \mathrm{rpm}$ (10\% reduction in speed, relative to control) and $3080 \mathrm{rpm}$ (20\% reduction in speed, relative to control). In order to assess the impact of centrifuge speed on cake odour, the digested sludge used in these trials was left untreated (i.e. no chemical addition except for polymer). In our laboratory trials, a $20 \%$ reduction in centrifuge speed $(3080 \mathrm{rpm})$ resulted in an approximate $30 \%$ decrease in the maximum TVOSC concentration, relative to the control. However, the solids content of the resulting cake was also significantly reduced from $15 \%$ at the control speed of $3850 \mathrm{rpm}$ to $10 \%$ at $3080 \mathrm{rpm}$, which would not be desirable from the point of view of WWTP operations.

\section{Analysis of OVACs in biosolids samples from the odour reduction trials}

No significant concentrations of OVACs were observed in any of the cake samples analysed during the monitoring period. In most cases the compounds were either at or below limits of quantification for the method. This is most likely because the monitoring period was not long enough for significant amounts of OVACs to form. Research has shown that indole and skatole begin to form at approximately 40 days, peak at approximately 100 days, and begin to disappear after about 125 - 135 days of incubation (Novak, 2012). The longest monitoring period over the course of the trials was 37 days.

\section{CONCLUSIONS}

In this study we identified some of the main odour compounds in biosolids samples obtained from a Western Australian WWTP and investigated chemical addition and reduction of centrifuge speed as 
potential odour reduction strategies. The main odour compounds identified in fresh biosolids samples from our test site included: DMS, DMDS and DMTS, while biosolids samples that were stored for several months contained indole and skatole.

Aluminium sulphate addition to digested sludge prior to dewatering offered the best odour reduction strategy amongst the options that were investigated, resulting in approximately $40 \%$ reduction in the maximum TVOSC concentration, relative to a control sample. From these preliminary trials, it appears that reduction of centrifuge speed would not be a viable odour reduction option for the test WWTP as it resulted in too much reduction in the solids content of the biosolids cake.

In most cases, results obtained from the HS SPME-GC-MS analyses were in general agreement with qualitative observations by a single trained odour assessor. In future studies, it would be beneficial to include dilution olfactometry measurements to obtain a more rigorous assessment of the overall odour generated from biosolids cake and to correlate/compare the results with measurements obtained using HS SPME-GC-MS.

While the investigations conducted in this Phase I study utilised sludge and biosolids samples from just one WWTP, Phase II studies will expand the scope to include biosolids and sludge sourced from different WWTPs. These Phase II studies will provide information on the odorous compounds present in biosolids produced at other WWTPs and whether the trialled odour reduction strategies are applicable to more than one type of wastewater treatment system.

\section{ACKNOWLEDGMENTS}

We thank the Water Corporation for their financial and technical support of this project. We also acknowledge the cooperation of the ChemCentre of Western Australia for allowing us the use of their laboratory centrifuge.

\section{REFERENCES}

Adams, G.A., Witherspoon, J., Erdal, Z., Forbes, B., McEwen, D., Hargreaves, R., Higgins, M.J. and Novak, J. (2008) Identifying and Controlling Odour in the Municipal Wastewater Environment Phase 3: Biosolids Processing Modifications for Cake Odour Reduction. Water Environment Research Foundation Report No 03-CTS-9T, Alexandria, VA, USA.

Chen, Y., Higgins, M., Murthy, S., Maas, N., Covert, K. and Toffey, W. (2006). Production of Odorous Indole, Skatole, $p$-Cresol, Toluene, Styrene and Ethylbenzene in Biosolids. Journal of Residuals Science \& Technology, 3, No. 4, 193-202.

Glindemann, D., Murthy, S.N., Higgins, M.J., Chen, Y.C. and Novak, J.T. (2006) Biosolids Incubation Method for Odorous Gas Measurement from Dewatered Sludge Cakes. Journal of Residuals Science and Technology, 3, No. 3, 153-160.

Gruchlik, Y., Heitz, A., Joll, C., Fouché L., Penney, N. and Charrois, J. (2012) Laboratory scale investigations of potential odour reduction strategies in biosolids. Water, 39, No. 7, 58-64.

Haberhauer-Troyer, C., Rosenberg, E. and Grasserbauer, M. (1999) Evaluation of Solid Phase Microextraction for Sampling of Volatile Organic Sulphur Compounds in Air for Subsequent Gas Chromatographic Analysis with Atomic Emission Detection. J. Chromatography A, 848, 305-315. 
Higgins, M.J., Chen, Y., Yarosz, D.P., Murthy, S.N., Maas, N.A., Glindemann, D. and Novak, J.T. (2006) Cycling of Volatile Organic Sulphur Compounds in Anaerobically Digested Biosolids and its Implications for Odours. Water Environ. Res., 78, 243-252.

Higgins, M.J., Chen, Y., Novak, J.T., Glindemann, D., Forbes, R., Erdal, Z., Witherspoon, J., McEwen, D., Murthy, S., Hargreaves, J.R. and Adams, G. (2008) A Multi-Plant Study to Understand the Chemicals and Process Parameters Associated with Biosolids Odours. Environmental Engineer: Applied Research and Practice, 5, 1-13. http://www.aaee.net/Downloads/EEJournalV5P1.pdf (accessed December 2012)

Higgins, M.J. (2010) Evaluation of aluminium and iron addition during conditioning and dewatering for odour control. Phase IV. Water Environment Research Foundation Report No 03-CTS-9A, Alexandria, VA, USA.

Kim, H., McConnell, L.L., Ramirez, M., Abu-Orf, M., Choi, H.L. and Peot, C. (2005) Characterization of Odours from Limed Biosolids Treated with Nitrate and Anthraquinone. Journal of Environmental Science and Health, Part A, 40, No. 1, 139-149.

Kristiana, I., Heitz, A., Joll, C., Sathasivan, A. (2010). Analysis of polysulfides in drinking water distribution systems using headspace solid-phase microextraction and gas chromatography-mass spectrometry. Journal of Chromatography A, 1217, 5995-6001.

Novak, J. T. and Park, C. (2010). Effect of aluminium and iron on odours, digestion efficiency and dewatering properties. Phase IV. Water Environment Research Foundation Report No 03-CTS-9b, Alexandria, VA, USA.

Novak, J. T. (2012). WERF ROSI Project: Regrowth, Odours and Sudden Increase (ROSI): Odours. WERF 2012 Webinar Series, 5 September 2012. www.werf.org/c/WebSeminars/9-5-12-ROSI.aspx (accessed 16 December 2012)

Subramanian, R., Novak, J.T., Murthy, S., Glindemann, D. and North, J. (2005) Investigating the Role of Process Conditions in Wastewater Sludge Odour Generation. In Proceedings of the Water Environment Federation, WEFTEC 2005, Washington DC, USA, 6582-6604.

Turkmen, M., Dentel, S.K., Chiu, P.C. and Hepner, S. (2004) Analysis of Sulphur and Nitrogen Odorants Using Solid Phase Microextraction and GC-MS. Water Science and Technology, 50, No. 4, 115-120. 